quantity of vapour of water than when the calomel is used alone.

The best apparatus for calomel fumigation hitherto made is, I believe, that of Messrs. Savigny and Co., of St. James's-street. This has been completed after many trials undertaken at the suggestion of Mr. Pollock, of St. George's Hospital. It consists of a kind of tin case, containing a spirit-lamp. In the centre, immediately over the wick of the lamp, is a small circular tin plate, upon which the ten or twenty grains of calomel are placed. Around this is a circular depression, which is half filled with boiling water. The patient places this on the ground, and sits over it, or near it, on a small cane stool. He is then enveloped, lamp and all, in a circular Mackintosh, which Messrs. Savigny have made for the purpose. When a Mackintosh cannot readily be procured, a double blanket answers the purpose very well. At the expiration of a quarter of an hour or twenty minutes, the calomel, the water, and the spirit in the lamp, will have disappeared, and the patient may then get into bed. It is well that a certain portion of the vapour should be inhaled during the bath. This will render the mercurial action more evident upon the patient's gums; but this is not always necessary for the cure of the disease. The length of time that the use of the baths should be continued, is probably about the same as would be required in other forms of mercurial treatment.

During the last eighteen months, I have employed this plan of treatment very extensively at the Lock Hospital, and there we seldom fail to get the patients' gums slightly affected in three or four days. The mercurial action may be regulated with the greatest nicety, either by the length of time the patient is exposed to the vapour of calomel, or by the quantity inhaled, or by the quantity used upon each occision. It is never requisite to produce salivation, and in the form now recommended any excessive mercurial action can scarcely be accidentally induced. The patients' constitutions are found, at the end of the mercurial course, to be in general as strong, and often much stronger, than when they commenced.

The conclusions which I would draw from the foregoing observations are, that in primary syphilis a large proportion of cases will not require any mercurial treatment at all; and that those which do may with tolerable certainty be distinguished from those which do not; that in those cases of primary syphilis which, if left to themselves, will infect the patient's system, mercurial treatment alone can be relied upon for preventing secondary symptoms; that in cases of secondary affections, although other medicines may remove the symptoms, mercury is much more effective than any other remedy in curing the disease so that the symptoms will not recur; and that of all the modes of administering mercury, mercurial fumigation is that which is attended with the least demand upon the powers of the patient's constitution; it is that which is regulated with the greatest facility, and that which can be maintained without inconvenience for the longest period; and I may add, that I believe it to be less frequently followed by a recurrence of secondary symptoms than any other mode of treatment whatever.

THE

\section{SPECULUM OCULI, OR OPHTHALMOSCOPE:}

ITS VALUE AS A DLAGNOSTIC AID IN THE EXPLORATION OF CERTAIN OBSCURE FORMS OF DISFASE AFFECTING THE EYE.

By JABEZ HOGG, Esq., M.R.C.S.E., ASSISTANT-SURGEON TO THE ROYAT WESTMINSTER OPHTHALMIC HOSPITAL. (Continued from $p$. 473.)

IF I may judge from the discussion which followed the reading of my paper on the Ophthalmoscope at the Medical Society of London, I should incline to the belief that, even at the present time, much difference of opinion prevails in this country with regard to the value of the instrument as a diagnostic aid.* This, donbtless, arises with most observers, who,

* "Dr. Jago, who has written on 'Ocular Spectres," is very sceptical as to the possibility of musc:e volitantes becoming visible to another person. $\mathbf{H e}$ more than hints that some of the ophthalmoscopists, who have described the 'shreds and flakes ' they have seen in patients' eyes, have unwittingly written down the wonders of their own visual organs. We will not dispute the possibility of such confusion of ownership when inexperienced persons are using the ophthlm sam w on taking it up, find it to be very difficult to project the reflected light into the eye, and at the same time to bring the fundus into focus. Then, again, the complications of some of the instruments invented and employed, increase the difficulties without offering any advantage over the simple instrument I have ventured to recommend. As with many other instraments, the more simple the more manageable it becomes:

Another and more serious source of failure is the pernicious use of atropine. I have repeatedly seen a drop of a weak solution of atropine produce in the healthy eye a very large amount of congestion of the retinoid vessels, more than sufficient to embarrass the diaonosis of any case, and which might well be mistaken for a diseased retina and choroid. On this account I have discontinued its use, and find no difficulty whatever in causing sufficient dilatation of the pupil, by simply placing my patient for a short time in is darkened room.

I often hear of great fatigue, or an agravation to the symptoms, occasioned by the large amount of light thrown on to disorganized or delicate tissues. This can only arise in careless or inexperienced hands, and may very properly be de signated an abuse of the instrument. Should an active inflammatory state of the iris or other membranes co-exist, the discomfit to the patient, produced by a very dull light, is quite sufficient to protect the organ, and prevent mischief. I firmly believe that we shall utimately find the ophthalmoscope take its place by the side of the stethoscope in our investigation of certain forms of disease.

My belief will, I am sure, derive greater value from a recital of cases lately, and at present, under treatment at the Royal Westminster Ophthalmic Hospital.

Fig. 5.-Right Eyye.

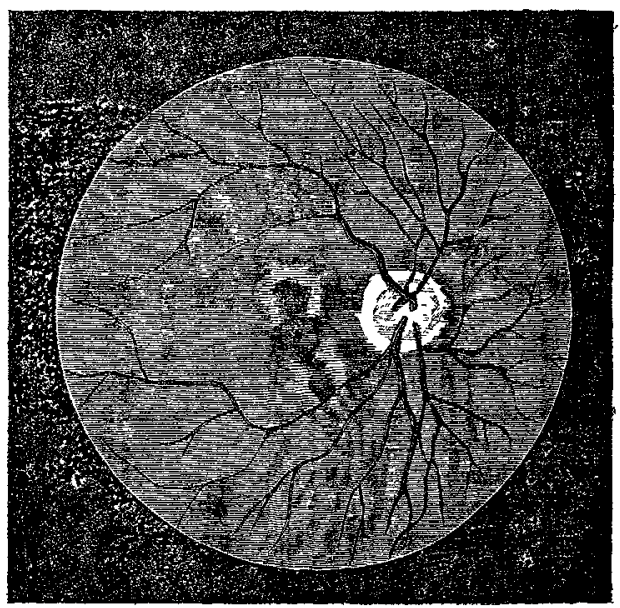

Hemiopy.-Insensibility of half the retina, with fixed black spot over the foramen centrale.

Fig. 6.-Left Eye.

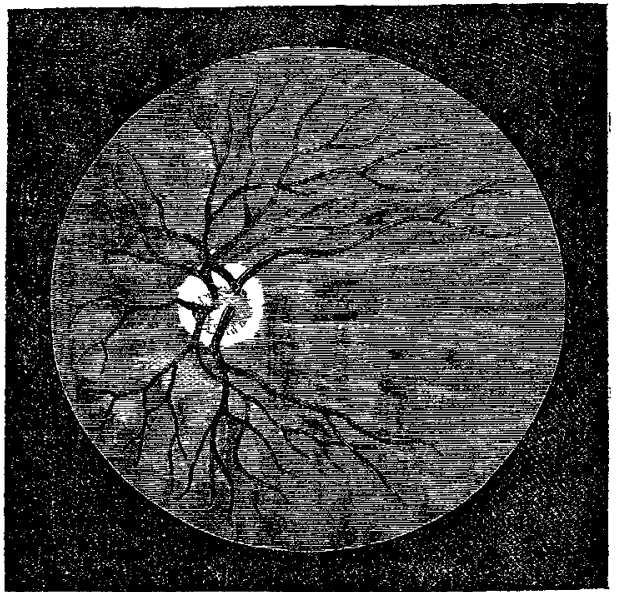

Hemiopy.-Insensibility of half the retina, with fixed blaek spot over the foramen centrale.

Hemiopy. - Insensibility of half the retina in both eyes, (Figs. 5 and 6.)-Filiza A-_, aged thirty-nine, married, has always used this instrument can most positively deny. "We suspect that the ophthalmoscope has not become familiar to Dr. Jaga. When it has, we be at prout he reject and at present he rejects, and will promote the degraded musca to the position MEDICO-CHIRURGICAL REVIEW, April, 185̃ 7, p. 421. 
had good health and sight until twelve years ago, when a rash broke out, which she said lookedlike measles, over her body; she had also a sore-throat, which was so bad as to prevent her swallowing, but does not remember having had any pain or redness in the eyes. She soon after discovered muscæ in her left eye, which moved up and down like little specks of soot, and found also, when reading, that the letters appeared to be on one side. A short time after, a semi-transparent blue veil, about the size of a shilling, appeared in the left eye, which partially obscured her sight when she looked at anything, but was not sufficiently dense to preclude her reading moderate-sized type. The sight of this eye has lately become worse, so that now on looking straight forwards she can see nothing immediately in the axis of vision, but anything situated at the outer side she can see just well enough to recognise. The right eye was healthy until three weeks since, when she felt some slight shooting pains through it, but did not discover any diminution of sight until three days ago (Dec. 19th), when she found it so dim as to prevent her reading the newspaper. This patient says she has had several miscarriages during the last twelve years, but has three children living who are healthy. Before the sight of her left eye failed she used to notice flashes of fire when closing her eyes to sleep. Examination by ophthalmoscope. - Left eye: cornea, lens,
and humours clear; entrance of the optic nerve has a brownish, dull-yellow tint over it, vessels are distinct, though small. Towards the position of the yellow spot is a patch of rather larger size than the optic nerve, the ground of which resembles that of the optic nerve; but, in several parts, fragments of brown and black pigment are to be noticed; the margin also of this patch appears of a deep brownish-red. There are also some bright-red streaks running parallel to one another at the lower part. The rest of the bottom of the eye has a brownish-red-cast; the retinoid vessels appear distinct though smaller than in a healthy eye. - Right eye: humours clear; entranoe of the optic nerve much the same as the left; the bottom of the eye appears of a brownish calour, but is streaked with white, and immediately over the position of the yellow spot itself, is a dark spot, nearly covering it. Below the horizon, the bottom of the eye has a dull, brownish-red cast; the retinoid vessels are small, but perfectly distinct, as in the left eye.

Dec. 30th, 1856. - She can read large print with the right eye if she looks down with the left eye; but says that when look. ing for a moment or two, a spot, the size of a threepenny piece, appears to come over the sight at the part she is looking at, which does not however entirely obscure the type, but renders it indistinct. States that she was subject to bleeding at the nose when she was suckling; and that when she menstruated, the bleeding was more severe. The epistaxis has ceased about twelve months. Bichloride of mercury and the decoction of cinchona bark mixture was prescribed for her. This she continued until January $16 \mathrm{th}, 1857$, when, a slight diarrhoea setting in, this was changed for small doses of mercury with chalk and rhubarb, every alternate night, and compound rhubarb mixture twice a day.

Feb. 2nd, 1857.--Improving; examined by ophthalmoscope; the black spot is not quite so large, but a very large quantity of pigment still obseures the retinal vessels in the left eye; the right is improving.

9th.-Examined by ophthalmoscope; right eye still improving; can see better; left optic nerve much clearer, and feel better if it were more natural." Ordered, compound iron mixture, one ounce, twice a day.

25th. - Improving in sight and general health. Since returaed to the country much improved.

CASE.-Musca Volitantes.-E. K- , female, aged fifty, admitted as an out-patient June 29,1855 . A delicate-looking person; pulse small, 60 ; has always had weak sight, and on several occasions, inflammation of the eyes; general health she considers to be good; married, and has several children. In 1853 her eyes became dim, and since then she has had a film floating about in the right eye; the dimness continued to increase up to the present time, and now vision is almost gone. The left eye is not yet in so bad a state, and sufficient vision remains to enable her to see her way about.

Examined by ophthalmoscope. - In the right eye masses of greyish streaks are floating about, the retinal vessels congested, irregular, and not well-detined; the capsule of the lens slightly thickened, especially at its periphery; the left eye is less congested, and apparently without floating masses. . Ordered a tonic mixture, containing soda, rhubarb, and infusion of quassia, with occasional and small doses of mercury pill. In a few weeks she was able to distinguish objects more clearly, and under a continuance of this treatment, the sight improved in the left eye; with the right eye she was able to read tolerably-sized print. The muscæ, or grey masses, became much less apparent, and in two months she left the hospital believing herself cured.

CASE.-A. B - aged twenty-nine, law clerk, applied June 29,1855 , with congestion of the retina of left eye. A thin, tall, light-complexioned, nervous man. Described the complaint as having, during several months, given him much, annoyance. At that time he could not see to read or write with it; general health tolerably good, but frequently suffering from bilious attacks. The iris of left eye slightly differs in colour; the lens, which appeared opaque by daylight, upon examination with the ophthalmoscope, was found not to be so, this colour evidently being due to congestion behind the lens; the vessels of the retina very irregular, and so much congested as to nearly conceal the entrance of the optic nerve; patches of serous effusion were seen between the choroid and retina; this eye only sensible to light at the inferior and internal portions. Mercurial pill, with aperient draughts, were ordered daily, under which treatment he much improved; tonics were then prescribed for a fortnight, and at the end of a month he was able to resume his occupation.

This case is valuable as showing the use of the ophthal. moscope, not only in deciding the question of opacity of the lens, but enabling us at once to direct attention to the seat of disease. Hence the treatment became most efficient. I have lately seen this patient, and he tells me he has had no return of the affection; but has been carefully following the advice given as regards resting the eyes as much as possible, and attending to his general health.

CASE. - R. R_, aged fifty-three, a cook, applied on 20th July, 1855. She states that her sight began to fail about two years ago, but has been able to do needlework until very recently; suffers from headaches and bilious attacks, which always increase the dulness of sight. General health, though not very good, has always permitted her to perform her daily work. Catamenia ceased ten years since. She now has a web over the right eye, which increases in size at night, and is accompanied by pain over the brow and head.

Examined by the ophthalmoscope.-The retinoid vessels much congested; a thick red web obscures the optic nerve. Left eye less congested than the right, and sight better in it; she indeed depends upon it for the small amount of useful vision remaining. A few grey striæ float about in the vitreous; lens quite clear.

Ordered a powder every night containing eight grains of calomel with five grains of Dover's powder, followed by an aperient draught every morning. Cantharides plaster to the neck. The effect of the mercury was kept up by a mixture of biniodide of mercury in decoction of bark; and at the end of two months she left the hospital, very much improved.

(To be continued.)

\section{EXPERIMENTS ON ARTIFICIAL DIGESTION.}

By THOS. K. CHAMBERS, M.D., F.R.C.P., LECTURER ON THE PRACIICE OF MEDTCINE AND PHYSICLAN TO ST. MARY'S HOSPITAL.

THE following experiments seem to exhibit pretty clearly the power of dissolving coagulated albumen possessed by different reagents employed for that purpose in artificial digestion. Pieces of hard-boiled white of egg were carefully wiped and weighed, and suspended without motion for twenty-four hours in beakers containing the solvent to be tested, mixed with about two ounces of water, and kept in a water oven at an even temperature. This of course does not exhibit a fair picture of the action of such reagents in the stomach, where the constant rolling movements, and the wiping away immediately of each successive portion dissolved by the copious stream of gastric juice, offer such favourable conditions. But it is a better comparative test than if the motion and stream are imitated artificially, as the attempt to do this acts un. equally on different specimens. I should advise all persons purchasing pepsine thus to estimate the value of the articles offered them. 